\title{
Optimality of block designs under the model with the first-order circular autoregression
}

\author{
Katarzyna Filipiak $^{1}$ (D) Razieh Khodsiani ${ }^{2} \cdot$ Augustyn Markiewicz $^{3}$
}

Received: 3 September 2018 / Revised: 16 December 2018 / Published online: 2 January 2019

(c) The Author(s) 2019

\begin{abstract}
In this paper optimal properties of some circular balanced block designs under the model with circular autoregression of order one are studied. Universal optimality of some balanced block designs with equal block sizes is proven and E-optimality of complete balanced block designs with the number of blocks equal to the number of treatments or the number of treatments reduced by two is shown.
\end{abstract}

Keywords Circular autoregression of order one - Circular balanced block design . Universal optimality $\cdot$ E-optimality $\cdot$ Nondirectionally neighbor balanced design

\section{Introduction}

The block experiments have been widely used in sciences, medical and engineering. The problem of universal optimality of block designs is widely studied for different correlation structures. Chai and Majumdar (2000) showed that under the nearest neighbor correlation structure the universally optimal block designs can be constructed from semibalanced arrays. Recently, Khodsiani and Pooladsaz (2017) characterized the universally optimal block designs under the hub correlation structure.

$凶 \quad$ Katarzyna Filipiak

Katarzyna.Filipiak@put.poznan.pl

Razieh Khodsiani

razieh.khodsiani@math.iut.ac.ir

Augustyn Markiewicz

amark@up.poznan.pl

1 Institute of Mathematics, Poznań University of Technology, Piotrowo 3A, 60-965 Poznan, Poland

2 Department of Mathematical Sciences, Isfahan University of Technology, Isfahan, 84156-83111, Iran

3 Department of Mathematical and Statistical Methods, Poznań University of Life Sciences, Wojska Polskiego 28, 60-637 Poznan, Poland 
Under the standard block model in which correlation between observations in every block decreases exponentially with the distance, the first-order linear autoregression covariance structure, $\operatorname{AR}(1, L)$, is considered in the literature. For some values of correlation parameter Kunert (1987) proved that the nearest neighbor balanced incomplete block designs, characterized by Gill and Shukla (1985), are universally optimal over the class of incomplete block designs. For positive correlations Pooladsaz and Martin (2005) extended the results of Kunert (1987) for the designs with block sizes exceeding the number of treatments. Kunert and Martin (1987b) extended the optimality results of some neighbor balanced designs for other autoregression correlation structures, such as e.g. circular autoregression $(\operatorname{AR}(1, C)$, defined in (2)) with positive correlation. Circular designs were considered for example by Rees (1967), in serological experiment in which treatments are arranged in circular blocks where every treatment has two neighbors. Another example is an experiment in marine biology in which five genotypes of bryozoan such that neighboring genotypes might interfere with each other were compared by suspending them in sea water around the circumference of a cylindrical tank; see Bayer and Todd (1996). One of the aims of this paper is to show universal optimality of some circular nondirectionally neighbor balanced designs under the model with $\operatorname{AR}(1, C)$ for positive and negative values of correlation coefficient.

Existence of universally optimal designs often has some limitations and for some combinations of design parameters the universally optimal designs can not exist. In such a case efficiency of some designs or optimality with respect to the specified criteria is considered. E-criterion is one of the most popular criteria that can be studied in this case, as it minimizes the largest variance among all best linear unbiased estimators of normalized linear contrasts, and plays an important role in e.g. admissibility investigations; cf. Pukelsheim (2006). Although determining the E-optimal designs is not easy especially when the observations are correlated because E-criterion is based on the eigenvalues of the information matrix of designs. Jacroux $(1982,1983)$ characterized E-optimal block designs for uncorrelated observations. Kunert and Martin (1987a) proved optimality of some neighbor designs with respect to the specific criteria, such as e.g. A-, D- or E-optimality under the model with observations correlated according to $\operatorname{AR}(1, L)$ with positive correlation parameter.

According to the circular structure of $\operatorname{AR}(1, C)$, optimality problem of block designs under this correlation structure is similar to the determining optimal designs in interference models. There are several results on optimality of circular neighbor balanced designs (CNBDs) and orthogonal arrays of type I under the fixed and mixed interference models, where the observations are correlated or not (see e.g. Druilhet 1999; Filipiak and Markiewicz 2003, 2004, 2005, 2007). Similarly as Kunert and Martin (1987a) we will consider circular complete block designs (known also as William's II(a) designs with extra plot). It is worth observing that designs with complete blocks are often used in practice. For example in UPOV (The International Union for the Protection of New Varieties of Plants) research, complete block designs are recommended in experiments when the number of treatments is less than 16. The designs with the same number of blocks as number of treatments and units are also applied in clinical trials. 
Since complete CNBDs that are universally optimal under the interference models exist only for specific combinations of design parameters, one can look for universally optimal designs for some other combinations of parameters or to determine designs which are optimal with respect to other criteria, e.g. E-optimality. Bailey et al. (2017) considered optimality of circular weakly neighbor balanced designs under the interference model. They showed the construction methods of such designs, mostly based on graph theory, and they applied Hamiltonian decomposition of matrices using GAP (2014) or Mathematica software. Nevertheless, numerical algorithms start to be not efficient for increasing number of treatments.

Regarding the circular block effects model with correlated errors and its connections to the interference model with uncorrelated errors, construction methods of E-optimal designs are even more complicated, and numerical algorithms fails even for relatively small number of treatments. Nevertheless, algebraic concepts of construction methods based on the left-neighboring matrix of a circular design being an incidence matrix of some particular graph, can be used to more general models.

This paper is organized as follows. Section 2 introduces notation and definitions. Section 3 presents some results about universal optimality of designs under the standard block model for any size of blocks when the observation errors are correlated according to $\operatorname{AR}(1, C)$. In Sect. 4 some E-optimal complete block designs are characterized.

\section{Notation and definitions}

Let us consider a set of circular block designs $\mathcal{D}_{t, b, k}$ where $t, b$ and $k$ are respectively the number of treatments, blocks and experimental units per block. A standard model associated with a design $d \in \mathcal{D}_{t, b, k}$ can be written as

$$
\mathbf{y}=\mu \mathbf{1}_{b k}+\mathbf{T}_{d} \boldsymbol{\tau}+\mathbf{B} \boldsymbol{\beta}+\boldsymbol{\varepsilon}
$$

where $\mu$ is a general mean, $\boldsymbol{\tau}$ and $\boldsymbol{\beta}$ are respectively the vectors of treatment and block effects, and $\boldsymbol{\varepsilon}$ is a vector of random errors with $\mathrm{E}(\boldsymbol{\varepsilon})=\mathbf{0}_{b k}$ and $\operatorname{Cov}(\boldsymbol{\varepsilon})=\sigma^{2} \mathbf{I}_{b} \otimes \mathbf{V}$. The $b k$ - vectors of ones and zeros are respectively denoted by $\mathbf{1}_{b k}$ and $\mathbf{0}_{b k}$, the identity matrix of order $b$ is denoted by $\mathbf{I}_{b}$, whilst $\sigma^{2}$ is a positive constant, $\mathbf{V}$ is a known, symmetric, positive definite covariance matrix and $\otimes$ denotes the Kronecker product. By $\mathbf{T}_{d}$ and $\mathbf{B}=\mathbf{I}_{b} \otimes \mathbf{1}_{k}$ respectively design matrices for treatments and blocks are denoted.

In this paper we are interested in characterization of universally optimal design. Thus, let us denote as $\mathbf{C}_{d}$ the information matrix of design $d$ for estimation of treatment effects in the model (1). Due to Kiefer (1975, Proposition 1), if $\mathbf{C}_{d} \mathbf{1}_{t}=\mathbf{0}_{t}$ for every $d \in \mathcal{D}_{t, b, k}$ and if design $d^{*}$ is such that $\mathbf{C}_{d^{*}}$ is completely symmetric and has maximal trace over $\mathcal{D}_{t, b, k}$, then $d^{*}$ is universally optimal among the class $\mathcal{D}_{t, b, k}$. Recall, that a $t \times t$ matrix $\mathbf{A}$ is completely symmetric, if all its diagonal elements are equal and all its off-diagonal elements are equal, that is $\mathbf{A}=(\alpha-\beta) \mathbf{I}_{t}+\beta \mathbf{1}_{t} \mathbf{1}_{t}^{\prime}, \alpha, \beta \in \mathbb{R}$.

For a design $d$ in $\mathcal{D}_{t, b, k}$ let $\lambda_{1}\left(\mathbf{C}_{d}\right) \geq \ldots \geq \lambda_{t-1}\left(\mathbf{C}_{d}\right)>\lambda_{t}\left(\mathbf{C}_{d}\right)=0$ be the eigenvalues of its information matrix. A design $d^{*} \in \mathcal{D}_{t, b, k}$ is called E-optimal over 
$\mathcal{D}_{t, b, k}$ if

$$
\lambda_{t-1}\left(\mathbf{C}_{d^{*}}\right) \geq \lambda_{t-1}\left(\mathbf{C}_{d}\right) \quad \text { for any } \quad d \in \mathcal{D}_{t, b, k} .
$$

The above E-optimality criterion can be also expressed in terms of variance, that is, E-optimal design minimizes the largest variance among all best linear unbiased estimators of normalized linear contrasts; cf. Pukelsheim (2006).

From Kiefer and Wynn (1981) the information matrix of design $d$ for estimation of treatment effects in the model (1) has the form

$$
\mathbf{C}_{d}=\mathbf{T}_{d}^{\prime}\left(\mathbf{I}_{b} \otimes \mathbf{V}^{*}\right) \mathbf{T}_{d}
$$

where $\mathbf{V}^{*}=\mathbf{V}^{-1}-\left(\mathbf{1}_{k}^{\prime} \mathbf{V}^{-1} \mathbf{1}_{k}\right)^{-1} \mathbf{V}^{-1} \mathbf{1}_{k} \mathbf{1}_{k}^{\prime} \mathbf{V}^{-1}$. Observe that since $\mathbf{1}_{b k}$ belongs to the column space of $\mathbf{T}_{d}$, we have $\mathbf{C}_{d} \mathbf{1}_{t}=\mathbf{0}_{t}$ and Kiefer's conditions for universal optimality can be applied.

We will assume that the observations in different blocks are uncorrelated but that observations within blocks are correlated according to a first-order circular autoregression process, $\operatorname{AR}(1, C)$, that is $\mathbf{V}=\left(v_{i j}\right), i, j=1, \ldots, k$, with

$$
v_{i j}= \begin{cases}\frac{\left(1+a^{k}\right)}{\left(1-a^{k}\right)\left(1-a^{2}\right)} & \text { if }|i-j|=0 \\ \frac{a^{h}+a^{k-h}}{1+a^{k}} & \text { if }|i-j|=h\end{cases}
$$

and $|a|<1$ being a correlation parameter.

Following Kunert and Martin (1987b) and Filipiak and Markiewicz (2005), the inverse of $\mathbf{V}$ can be presented as

$$
\mathbf{V}^{-1}=\left(1+a^{2}\right) \mathbf{I}_{k}-a\left(\mathbf{H}_{k}+\mathbf{H}_{k}^{\prime}\right)
$$

where $\mathbf{H}_{k}$ denotes the $k \times k$ left-neighbor incidence matrix, that is the matrix with $(i, j)$ th element equal to 1 if $i-j=1$ and $h_{1, k}=1$, and 0 otherwise. Hence

$$
\mathbf{V}^{*}=\left(1+a^{2}\right) \mathbf{I}_{k}-a\left(\mathbf{H}_{k}+\mathbf{H}_{k}^{\prime}\right)-\frac{(1-a)^{2}}{k} \mathbf{1}_{k} \mathbf{1}_{k}^{\prime}
$$

and the information matrix can be written as

$$
\mathbf{C}_{d}=\left(1+a^{2}\right) \mathbf{R}_{d}-a\left(\mathbf{S}_{d}+\mathbf{S}_{d}^{\prime}\right)-\frac{(1-a)^{2}}{k} \mathbf{N}_{d} \mathbf{N}_{d}^{\prime}
$$

where $\mathbf{R}_{d}=\mathbf{T}_{d}^{\prime} \mathbf{T}_{d}$ is a diagonal matrix with replications of $i$ th treatment on the diagonal, $\mathbf{S}_{d}=\mathbf{T}_{d}^{\prime}\left(\mathbf{I}_{t} \otimes \mathbf{H}_{k}\right) \mathbf{T}_{d}$ is a $t \times t$ left-neighboring matrix of a circular design $d$ (cf. Filipiak et al. 2008) and $\mathbf{N}_{d}=\mathbf{T}_{d}^{\prime} \mathbf{B}$ is the treatment-block incidence matrix. Recall, that the design is called circular if each block of a design has the form of a circle or, if plots in blocks are arranged in linear forms, but there are additional border plots at the beginning of each block, containing the same treatment as at the opposite end of the block (cf. Druilhet 1999). The border plots receive treatments but are not used for measuring the response variables. Note that the $(i, j)$-th element of $\mathbf{S}_{d}, s_{d, i j}$, 
denotes the number of occurrences of treatment $i$ with treatment $j$ as a left neighbor. If a design $d$ has no self-neighbors, the diagonal entries of $\mathbf{S}_{d}$ are equal to zero. Since in our considerations the matrix $\mathbf{S}_{d}+\mathbf{S}_{d}^{\prime}$ plays a crucial role we denote its elements by $\xi_{i j}$, that is $\xi_{i j}=s_{d, i j}+s_{d, j i}$. It is clear that for designs without self-neighbors $\xi_{i i}=0$.

Throughout this paper we use some properties of a balanced block design (BBD), i.e. such a design $d \in \mathcal{D}_{t, b, k}$ for which (i) all $n_{d, i j}=\lfloor k / t\rfloor$ or $\lfloor k / t\rfloor+1$, (ii) all $r_{d, i}$ are equal (say $r$ ), and (iii) every pair of distinct treatments occurs together in the same number of blocks ( number of replications of the $i$ th treatment in $d$, the $i$ th diagonal entry of $\mathbf{R}_{d}$, and $n_{d, i j}$ is the $(i, j)$ th entry $\mathbf{N}_{d}$ (cf. Kiefer 1958). A BBD reduces to a balanced incomplete block design (BIBD) when $k<t$. All designs satisfying (i) are called generalized binary designs (Das and Dey 1989), while designs satisfying (ii) are called equireplicated designs. The class of generalized binary designs with $k \leq t$ will be denoted by $\mathcal{B}_{t, b, k}$.

The following algebraic notation and definitions is used in Sect. 4 of the paper.

An $n \times n$ matrix $\mathbf{A}$ is said to be reducible if either of the following conditions is satisfied:

(a) $n=1$ and $\mathbf{A}=0$;

(b) $n \geq 2$ and there is a permutation matrix $\mathbf{P} \in \mathcal{P}_{n}$ and an integer $u$ with $1 \leq$ $u \leq n-1$ such that $\mathbf{P}^{\prime} \mathbf{A P}=\left(\begin{array}{cc}\mathbf{B}_{11} & \mathbf{B}_{12} \\ \boldsymbol{\Theta} & \mathbf{B}_{22}\end{array}\right)$ where $\mathbf{B}_{11} \in \mathbb{R}^{u \times u}, \mathbf{B}_{12} \in \mathbb{R}^{u \times(n-u)}$, $\mathbf{B}_{22} \in \mathbb{R}^{(n-u) \times(n-u)}$ and $\boldsymbol{\Theta}$ is an $(n-u) \times u$ zero matrix.

A matrix is called irreducible if it is not reducible.

Let $\mathcal{P}_{n}$ be the set of all permutation matrices of order $n$ and let $\overline{\mathcal{P}}_{n} \subset \mathcal{P}_{n}$ be the set of permutation matrices with zero diagonal (the set of derangement matrices of order $n$ ). The matrix $\mathbf{P}^{\prime} \mathbf{A P}$, where $\mathbf{P} \in \mathcal{P}_{n}$, is called permutationally similar to $\mathbf{A}$. It is worth noting that the eigenvalues of $\mathbf{A}$ and a matrix permutationally similar to $\mathbf{A}$ are the same.

\section{Universal optimality over the class $\mathcal{D}_{t, b, k}$}

If $a=0$ there is no correlation structure in model (1) and it is known that every BBD is universally optimal over $\mathcal{D}_{t, b, k}$ (cf. Shah and Sinha 1989). In this section we characterize universally optimal designs under model (1) with $\operatorname{AR}(1, C)$ structure, where $a \in(-1,1) \backslash\{0\}$. We denote $\overline{\mathcal{D}}_{t, b, k}$ as the subclass of $\mathcal{D}_{t, b, k}$ with no treatment preceded by itself.

Theorem 1 The circular BBD $d^{*}$ such that $\mathbf{S}_{d^{*}}+\mathbf{S}_{d^{*}}^{\prime}$ is completely symmetric with zero diagonal, is universally optimal under model (1) over the class $\mathcal{D}_{t, b, k}$ if a $>0$, and over the class $\overline{\mathcal{D}}_{t, b, k}$ if $a<0$.

Proof By (3) for the circular BBD $d^{*}$ such that $\mathbf{S}_{d^{*}}+\mathbf{S}_{d^{*}}^{\prime}$ is completely symmetric,

$$
\mathbf{C}_{d^{*}}=\left[r\left(1+a^{2}\right)-\frac{(1-a)^{2}}{k}(r-\lambda)\right] \mathbf{I}_{t}-a\left(\mathbf{S}_{d^{*}}+\mathbf{S}_{d^{*}}^{\prime}\right)-\frac{\lambda(1-a)^{2}}{k} \mathbf{1}_{t} \mathbf{1}_{t}^{\prime}
$$


Thus, $\mathbf{C}_{d^{*}}$ is completely symmetric and since $\operatorname{tr}\left(\mathbf{S}_{d^{*}}+\mathbf{S}_{d^{*}}^{\prime}\right)=0$ we obtain

$$
\operatorname{tr}_{d^{*}}=b k\left[\left(1+a^{2}\right)-\frac{(1-a)^{2}}{k}\right] .
$$

Let $d$ be a block design in $\mathcal{D}_{t, b, k}$. By (3) we have,

$$
\operatorname{tr}_{d}=b k\left(1+a^{2}\right)-2 a \operatorname{tr} \mathbf{S}_{d}-\frac{(1-a)^{2}}{k} \operatorname{tr}\left(\mathbf{N}_{d} \mathbf{N}_{d}^{\prime}\right)
$$

It is known (cf. Shah and Sinha 1989) that $\operatorname{tr}\left(\mathbf{N}_{d} \mathbf{N}_{d}^{\prime}\right)$ is minimal for BBD. Thus, it is immediately seen that $\operatorname{tr} \mathbf{C}_{d^{*}}$ is maximal for $d^{*}$ being BBD over the class $\mathcal{D}_{t, b, k}$ if $a>0$ and over the class $\overline{\mathcal{D}}_{t, b, k}$ if $a<0$.

It is worth noting that for $a=0$ and $d \in \mathcal{D}_{t, b, k} \backslash \mathcal{B}_{t, b, k}, \operatorname{tr} \mathbf{C}_{\mathrm{BBD}}>\operatorname{tr} \mathbf{C}_{d}$. Moreover, for $k \leq t, \operatorname{tr} \mathbf{S}_{\mathrm{BBD}}=0$, and from continuity of the trace (as a function of $a$ ) it follows that for negative $a$, sufficiently close to 0 , the BBD is universally optimal over the class $\mathcal{D}_{t, b, k}$. Moreover, for small number of treatments (e.g. $t=k=3,4,5$ ), the BBDs are universally optimal over the class $\mathcal{D}_{t, b, k}$ even for all negative $a$.

One of the example of a designs satisfying conditions of Theorem 1 are nondirectionally neighbor balanced designs (NdNBD), that is designs with $\mathbf{S}_{d}+\mathbf{S}_{d}^{\prime}=\mathbf{1}_{t} \mathbf{1}_{t}^{\prime}-\mathbf{I}_{t}$ (cf. Azaiis et al. 1993). However, NdNBD cannot exist for even $t$. Nevertheless, we can consider generalized NdNBDs having the property of $\mathbf{S}_{d}+\mathbf{S}_{d}^{\prime}$ being proportional to $\mathbf{1}_{t} \mathbf{1}_{t}^{\prime}-\mathbf{I}_{t}$, which satisfies Theorem 1. For example the following design with blocks represented as rows

$$
d^{*}=\left(\begin{array}{ccc}
1 & 2 & 3 \\
1 & 4 & 5 \\
1 & 2 & 6 \\
1 & 4 & 6 \\
1 & 3 & 5 \\
2 & 4 & 3 \\
2 & 5 & 6 \\
2 & 4 & 5 \\
3 & 4 & 6 \\
3 & 5 & 6
\end{array}\right) \text { with } \mathbf{S}_{d^{*}}+\mathbf{S}_{d^{*}}^{\prime}=2\left(\mathbf{1}_{6} \mathbf{1}_{6}^{\prime}-\mathbf{I}_{6}\right)
$$

is a generalized NdNBD. Observe moreover, that CNBDs and circular neighbor balanced designs at distance 2 (CNBD2) defined e.g. by Druilhet (1999), that can exist also for even $t$, are also generalized NdNBDs. Their construction methods for complete blocks and blocks of size $t-1$ can be found in Azaïs et al. (1993).

Universal optimality of CNBD2 under the wider model with $\mathrm{AR}(1, C)$, namely the one-sided interference model, was shown in Filipiak and Markiewicz (2005). 


\section{E-optimal complete block designs for $b=t-2$ and $b=t$}

In this section we assume $t \geq 3$. We denote the subclass of equireplicated designs of $\mathcal{D}_{t, b, k}$ by $\mathcal{R}_{t, b, k}$ and $\overline{\mathcal{R}}_{t, b, k}$ consists of the equireplicated designs with no treatment preceded by itself.

Assume that $d \in \mathcal{B}_{t, b, t}$. Since the blocks of designs are complete now, that is $k=t$, every binary design is a BBD. Thus, due to (4),

$$
\mathbf{C}_{d}=b\left(1+a^{2}\right) \mathbf{I}_{t}-\frac{b(1-a)^{2}}{t} \mathbf{1}_{t} \mathbf{1}_{t}^{\prime}-a\left(\mathbf{S}_{d}+\mathbf{S}_{d}^{\prime}\right)
$$

and $\mathbf{S}_{d} \mathbf{1}_{k}=\mathbf{S}_{d}^{\prime} \mathbf{1}_{k}=b \mathbf{1}_{k}$. Hence $\lambda_{t}\left(\mathbf{C}_{d}\right)=0$ and, for $i=1, \ldots, t-1$,

$$
\lambda_{i}\left(\mathbf{C}_{d}\right)=b\left(1+a^{2}\right)+\lambda_{i}\left(-a\left(\mathbf{S}_{d}+\mathbf{S}_{d}^{\prime}\right)\right) .
$$

and it is enough to find minimum of $\lambda_{2}\left(\mathbf{S}_{d}+\mathbf{S}_{d}^{\prime}\right)$ over $d \in \mathcal{B}_{t, b, t}$ if $a>0$, and maximum of $\lambda_{t}\left(\mathbf{S}_{d}+\mathbf{S}_{d}^{\prime}\right)$ over $d \in \mathcal{B}_{t, b, t}$ if $a<0$.

Observe that every irreducible derangement matrix is permutationally similar to the incidence matrix $\mathbf{H}_{n}$ defined in Sect. 2. From circularity of $\mathbf{H}_{n}$ it follows, that the non-ordered eigenvalues $\mu_{i}\left(\mathbf{H}_{n}\right), i=1,2, \ldots, n$, are equal to the roots of unity, that is $\mu_{i}\left(\mathbf{H}_{n}\right)=\omega_{i-1}$. Hence,

$$
\mu_{i}\left(\mathbf{H}_{n}+\mathbf{H}_{n}^{\prime}\right)=\mu_{i}\left(\mathbf{H}_{n}+\mathbf{H}_{n}^{-1}\right)=2 \cos \frac{2 \pi(i-1)}{n}
$$

and

$$
\begin{aligned}
& \lambda_{1}\left(\mathbf{H}_{n}+\mathbf{H}_{n}^{\prime}\right)=2 \\
& \lambda_{n}\left(\mathbf{H}_{n}+\mathbf{H}_{n}^{\prime}\right)=\left\{\begin{array}{lr}
-2 & \text { for even } n \\
2 \cos \frac{(n \pm 1) \pi}{n} \rightarrow-2^{+} & \text {for odd } n
\end{array}\right.
\end{aligned}
$$

Moreover, since every reducible derangement matrix is permutationally similar to the block-diagonal matrix with $\mathbf{H}_{n_{j}}$ on the diagonal, $\sum_{j=1}^{u} n_{j}=n, 1<u \leq n$, the set of its eigenvalues consists of the $n_{j}$ th roots of unity, $j=1, \ldots, u$.

Throughout the paper the following inequalities for the eigenvalues of a diagonal $l \times l$ block, say $\mathbf{A}_{11}$, of partitioned matrix $\mathbf{A}$ :

$$
\begin{aligned}
\lambda_{n-m+1}(\mathbf{A}) & \leq \lambda_{l-m+1}\left(\mathbf{A}_{11}\right) \\
\lambda_{m}(\mathbf{A}) & \geq \lambda_{m}\left(\mathbf{A}_{11}\right)
\end{aligned}
$$

where $m=1,2, \ldots, l$; cf. Marshall et al. (2011).

The following lemmas will be useful for characterization of E-optimal designs.

Lemma 1 If the derangement matrix $\mathbf{P} \in \overline{\mathcal{P}}_{n}$ is permutationally similar to the matrix

(i) $\mathbf{H}_{n}$ if $n=2,7$; 
(ii) $\mathbf{I}_{2} \otimes \mathbf{H}_{2}$ or $\mathbf{H}_{4}$ if $n=4$;

(iii) $\mathbf{I}_{m} \otimes \mathbf{H}_{3}$ if $n=3 m, m \in \mathbb{N}$;

(iv) $\operatorname{diag}\left(\mathbf{I}_{i} \otimes \mathbf{H}_{3}, \mathbf{I}_{j} \otimes \mathbf{H}_{5}\right)$ if $n=5$ or $n \geq 8$ and $n \neq 3 m, m \in \mathbb{N}$ with $n=3 i+5 j$ for some $i \in \mathbb{N} \cup\{0\}$ and $j \in \mathbb{N}$,

then the minimal eigenvalue of $\mathbf{P}+\mathbf{P}^{\prime}$, that is $\lambda_{n}\left(\mathbf{P}+\mathbf{P}^{\prime}\right)$, is maximal over $\overline{\mathcal{P}}_{n}$.

Proof From (6) the maximum of $\lambda_{n}\left(\mathbf{P}+\mathbf{P}^{\prime}\right)$ is obtained for $\mathbf{P} \in \overline{\mathcal{P}}_{n}$ permutationally similar to the block-diagonal matrix with irreducible diagonal blocks of odd order as small as possible. Since $n=5$ and every $n \geq 8$ can be presented as $3 i+5 j$ for some $i, j \in \mathbb{N} \cup\{0\}$, we obtain (iii) and (iv). Moreover, for $n=3 i+5 j, j \neq 0$, the maximum of $\lambda_{n}\left(\mathbf{P}+\mathbf{P}^{\prime}\right)$ is equal to the maximum of $\lambda_{n}\left(\mathbf{H}_{5}+\mathbf{H}_{5}^{\prime}\right)$, that is $2 \cos (4 \pi / 5)$.

If $n=2$ or 4 then any decomposition of $n$ for odd numbers is possible and hence $\lambda_{n}\left(\mathbf{P}+\mathbf{P}^{\prime}\right)=-2$. Similarly, $n=7$ cannot be decomposed for odd numbers and hence $\max \lambda_{7}\left(\mathbf{P}+\mathbf{P}^{\prime}\right)=\lambda_{7}\left(\mathbf{H}_{7}+\mathbf{H}_{7}^{\prime}\right)=2 \cos (6 \pi / 7)$. $\mathbf{P} \in \overline{\mathcal{P}}_{7}$

Lemma 2 If the derangement matrix $\mathbf{P} \in \overline{\mathcal{P}}_{n}$ is permutationally similar to $\mathbf{H}_{n}$, then the second maximal eigenvalue of $\mathbf{P}+\mathbf{P}^{\prime}$, that is $\lambda_{2}\left(\mathbf{P}+\mathbf{P}^{\prime}\right)$, is minimal over $\overline{\mathcal{P}}_{n}$.

Proof From (5), $\lambda_{1}\left(\mathbf{H}_{n}+\mathbf{H}_{n}^{\prime}\right)=2$ has multiplicity 1. For arbitrary reducible $\mathbf{P} \in \overline{\mathcal{P}}_{n}$ permutationally similar to $\operatorname{diag}\left(\mathbf{H}_{n_{1}}, \ldots, \mathbf{H}_{n_{u}}\right)$, the largest eigenvalue $\lambda_{1}\left(\mathbf{P}+\mathbf{P}^{\prime}\right)=2$ has multiplicity $u$. Thus, the matrix $\mathbf{P} \in \overline{\mathcal{P}}_{n}$ which minimizes $\lambda_{2}\left(\mathbf{P}+\mathbf{P}^{\prime}\right)$ has to be irreducible.

Similar results as in Lemmas 1 and 2 can be found in Filipiak et al. (2008).

\subsection{E-optimal complete block designs for $b=t-2$}

Let define the following subclass of $\mathcal{B}_{t, t-2, t}$ :

$$
\widetilde{\mathcal{B}}_{t, t-2, t}=\left\{d: \mathbf{S}_{d}=\mathbf{1}_{t} \mathbf{1}_{t}^{\prime}-\mathbf{I}_{t}-\mathbf{P}_{d}, \mathbf{P}_{d} \in \overline{\mathbf{P}}_{t}\right\}
$$

Then, for every $d \in \widetilde{\mathcal{B}}_{t, t-2, t}$ the information matrix has the form

$$
\mathbf{C}_{d}=\left[(t-2)\left(1+a^{2}\right)+2 a\right] \mathbf{I}_{t}-\beta \mathbf{1}_{t} \mathbf{1}_{t}^{\prime}+a\left(\mathbf{P}_{d}+\mathbf{P}_{d}^{\prime}\right)
$$

with $\beta=2 a+\frac{(t-2)(1-a)^{2}}{t}$, and $\beta>0$ for every $|a|<1$. Thus, to determine E-optimal design over $\widetilde{\mathcal{B}}_{t, t-2, t}$ it is enough to find a design $d^{*}$ such that for any $d \in \widetilde{\mathcal{B}}_{t, t-2, t}$

$$
\begin{aligned}
& \lambda_{t}\left(\mathbf{P}_{d^{*}}+\mathbf{P}_{d^{*}}^{\prime}\right) \geq \lambda_{t}\left(\mathbf{P}_{d}+\mathbf{P}_{d}^{\prime}\right) \text { if } a>0 \\
& \lambda_{2}\left(\mathbf{P}_{d^{*}}+\mathbf{P}_{d^{*}}^{\prime}\right) \leq \lambda_{2}\left(\mathbf{P}_{d}+\mathbf{P}_{d}^{\prime}\right) \text { if } a<0 .
\end{aligned}
$$

We prove the following theorem.

Theorem 2 If there exists design $d^{*}$ with $\mathbf{S}_{d^{*}}=\mathbf{1}_{t} \mathbf{1}_{t}^{\prime}-\mathbf{I}_{t}-\mathbf{P}_{d^{*}}$ such that $\mathbf{P}_{d^{*}}$ is permutationally similar to the matrix 
(i) $\mathbf{H}_{t}$ if $t=2,7$;

(ii) $\mathbf{I}_{2} \otimes \mathbf{H}_{2}$ or $\mathbf{H}_{4}$ if $t=4$;

(iii) $\mathbf{I}_{m} \otimes \mathbf{H}_{3}$ if $t=3 m, m \in \mathbb{N}$;

(iv) $\operatorname{diag}\left(\mathbf{I}_{i} \otimes \mathbf{H}_{3}, \mathbf{I}_{j} \otimes \mathbf{H}_{5}\right)$ if $t=5$ or $t \geq 8$ and $t \neq 3 m, m \in \mathbb{N}$ with $t=3 i+5 j$ for some $i \in \mathbb{N} \cup\{0\}$ and $j \in \mathbb{N}$;

then $d^{*}$ is E-optimal under model (1) with $A R(1, C)$ and a $>0$ over the class $\overline{\mathcal{R}}_{t, t-2, t}$.

Proof Let $a>0$. We prove the thesis in three steps.

Step 1. Let $d \in \widetilde{\mathcal{B}}_{t, t-2, t}$. We obtain the thesis by condition (9) and Lemma 1.

Step 2. Let $d \in \mathcal{B}_{t, t-2, t} \backslash \widetilde{\mathcal{B}}_{t, t-2, t}$. We have to show that

$$
\lambda_{2}\left(\mathbf{S}_{d^{*}}+\mathbf{S}_{d^{*}}^{\prime}\right) \leq \lambda_{2}\left(\mathbf{S}_{d}+\mathbf{S}_{d}^{\prime}\right)
$$

From nonnegative definiteness of $\mathbf{C}_{d}$ it is known that $\lambda_{1}\left(\mathbf{S}_{d}+\mathbf{S}_{d}^{\prime}\right)=2 b$ and $\mathbf{S}_{d}+\mathbf{S}_{d}^{\prime}$ has positive and negative eigenvalues. Observe, that one of the eigenvalues of $\mathbf{S}_{d}+$ $\mathbf{S}_{d}^{\prime}-\frac{2 b}{t} \mathbf{1}_{t} \mathbf{1}_{t}^{\prime}$ is equal to zero. For convenience we will study the properties of the matrix $\mathbf{G}_{d}=2 b \mathbf{1}_{t} \mathbf{1}_{t}^{\prime}-t\left(\mathbf{S}_{d}+\mathbf{S}_{d}^{\prime}\right)$, also with zero eigenvalue, for which condition (11) is equivalent to

$$
\lambda_{t-1}\left(\mathbf{G}_{d^{*}}\right) \geq \lambda_{t-1}\left(\mathbf{G}_{d}\right) .
$$

Note that $\lambda_{t-1}\left(\mathbf{G}_{d^{*}}\right) \geq 0$. Since the diagonal entries of $\mathbf{G}_{d}$ are equal to $2(t-2)$ and the off-diagonal entries, $g_{d, i j}$, are of the form $2(t-2)+t \xi_{i j}$ with $\xi_{i j} \in\{0,1, \ldots, t-2\}$, we will consider several cases of $\mathbf{G}_{d}$.

(a) Let $\xi_{i j} \leq 2$ for every $i \neq j$. Then, there exists a design $\widetilde{d} \in \widetilde{\mathcal{B}}_{t, t-2, t}$ such that $\mathbf{S}_{d}+\mathbf{S}_{d}^{\prime}=\mathbf{S}_{\tilde{d}}+\mathbf{S}_{\tilde{d}}^{\prime}$ and the thesis follows from Lemma 1.

(b) Assume now that $\xi_{i j} \in\{0,1,2,3\}$ for every $i \neq j$ and there exist at least one $i$ and $j$ such that $\xi_{i j}=3$. We consider four cases separately.

(b1) Let $t \leq 7$. Then there exists a submatrix of $\mathbf{G}_{d}$ of the form

$$
\mathbf{M}^{(1)}=2(t-2) \mathbf{1}_{2} \mathbf{1}_{2}^{\prime}-t\left(\begin{array}{ll}
0 & 3 \\
3 & 0
\end{array}\right)
$$

such that $\operatorname{det}\left(\mathbf{M}^{(1)}\right)=3 t(t-8)<0$. From $(7) \lambda_{t}\left(\mathbf{G}_{d}\right) \leq \lambda_{2}\left(\mathbf{M}^{(1)}\right)<0$. Since additionally $\mathbf{G}_{d}$ is singular, $\lambda_{t-1}\left(\mathbf{G}_{d}\right) \leq 0$ and (12) is satisfied trivially.

(b2) Let $t \geq 8$ and there exists at least one $i$ and $j, i \neq j$, such that $\xi_{i j}=0$. Then we can find a submatrix of $\mathbf{G}_{d}$ of the form

$$
\mathbf{M}^{(2)}=2(t-2) \mathbf{1}_{3} \mathbf{1}_{3}^{\prime}-t\left(\begin{array}{ccc}
0 & 0 & \xi_{i j} \\
0 & 0 & \xi_{i^{\prime} j^{\prime}} \\
\xi_{j i} & \xi_{j^{\prime} i^{\prime}} & 0
\end{array}\right)
$$

for which $\operatorname{det}\left(\mathbf{M}^{(2)}\right)=-2\left(\xi_{i j}-\xi_{i^{\prime} j^{\prime}}\right)^{2} t^{2}(t-2) \leq 0$ for every $t \geq 3$ and $\xi_{i j}, \xi_{i^{\prime} j^{\prime}} \in\{0,1,2,3\}$. Thus, $\lambda_{3}\left(\mathbf{M}^{(2)}\right)<0$ (also in the case $\xi_{i j}=\xi_{i^{\prime} j^{\prime}}$, for which additionally $\left.\lambda_{2}\left(\mathbf{M}^{(2)}\right)=0\right)$. From (7) $\lambda_{t}\left(\mathbf{G}_{d}\right) \leq \lambda_{3}\left(\mathbf{M}^{(2)}\right)<0$. Since additionally one of the eigenvalue of $\mathbf{G}_{d}$ is equal to zero, $\lambda_{t-1}\left(\mathbf{G}_{d}\right) \leq 0$ and (12) is satisfied trivially. 
(b3) Let $t \geq 8$ and there exist exactly one $i \neq j$ such that $\xi_{i j}=3$, and for every $i \neq j$ we have $\xi_{i j} \neq 0$. Then we can find one of the submatrices of $\mathbf{G}_{d}$ of the form:

$$
\begin{aligned}
& \mathbf{M}^{(3)}=2(t-2) \mathbf{1}_{3} \mathbf{1}_{3}^{\prime}-t\left(\begin{array}{lll}
0 & 3 & 1 \\
3 & 0 & 1 \\
1 & 1 & 0
\end{array}\right), \\
& \mathbf{M}^{(4)}=2(t-2) \mathbf{1}_{6} \mathbf{1}_{6}^{\prime}-t\left(\begin{array}{llllll}
0 & 3 & 1 & 1 & 2 & 2 \\
3 & 0 & 2 & 2 & 1 & 1 \\
1 & 2 & 0 & 2 & 2 & 2 \\
1 & 2 & 2 & 0 & 2 & 2 \\
2 & 1 & 2 & 2 & 0 & 2 \\
2 & 1 & 2 & 2 & 2 & 0
\end{array}\right) \\
& =2(t-2) \mathbf{1}_{6} \mathbf{1}_{6}^{\prime}-t \mathbf{V}^{(4)} \text {, } \\
& \mathbf{M}^{(5)}=2(t-2) \mathbf{1}_{8} \mathbf{1}_{8}^{\prime}-t\left(\begin{array}{cccccccc}
0 & 3 & 2 & 2 & 2 & 1 & 1 & 1 \\
3 & 0 & 1 & 1 & 1 & 2 & 2 & 2 \\
2 & 1 & 0 & 1 & 2 & 2 & 2 & 2 \\
2 & 1 & 1 & 0 & 2 & 2 & 2 & 2 \\
2 & 1 & 2 & 2 & 0 & 1 & 2 & 2 \\
1 & 2 & 2 & 2 & 1 & 0 & 2 & 2 \\
1 & 2 & 2 & 2 & 2 & 2 & 0 & 1 \\
1 & 2 & 2 & 2 & 2 & 2 & 1 & 0
\end{array}\right) \\
& =2(t-2) \mathbf{1}_{8} \mathbf{1}_{8}^{\prime}-t \mathbf{V}^{(5)} \text {. }
\end{aligned}
$$

It is easy to calculate that $\operatorname{det}\left(\mathbf{M}^{(3)}\right)=-12 t^{2}<0$ for every $t$. From (7) $\lambda_{t}\left(\mathbf{G}_{d}\right) \leq \lambda_{3}\left(\mathbf{M}^{(3)}\right)<0$. Since additionally $\mathbf{G}_{d}$ is singular, $\lambda_{t-1}\left(\mathbf{G}_{d}\right) \leq 0$ and (12) is satisfied trivially.

In two remaining cases it can be calculated that $\mathbf{V}^{(u)}, u=4,5$, is singular with $\lambda_{2}\left(\mathbf{V}^{(u)}\right)=0$. From (8) $\lambda_{2}\left(\mathbf{S}_{d}+\mathbf{S}_{d}^{\prime}\right) \geq \lambda_{2}\left(\mathbf{V}^{(u)}\right)=0$ and condition (11) is satisfied trivially.

(b4) Let $t \geq 8$ and there exist at least two pairs of indices $\left(i^{\prime}, j^{\prime}\right) \neq\left(i^{\prime \prime}, j^{\prime \prime}\right)$, such that $\xi_{i^{\prime} j^{\prime}}=\xi_{i^{\prime \prime} j^{\prime \prime}}=3$, and for every $i \neq j$ we have $\xi_{i j} \neq 0$. Then we can find one of the submatrices of $\mathbf{G}_{d}$ of the form:

$$
\begin{aligned}
& \mathbf{M}^{(6)}=2(t-2) \mathbf{1}_{4} \mathbf{1}_{4}^{\prime}-t\left(\begin{array}{llll}
0 & 3 & 1 & 1 \\
3 & 0 & 2 & 2 \\
1 & 2 & 0 & 3 \\
1 & 2 & 3 & 0
\end{array}\right)=2(t-2) \mathbf{1}_{4} \mathbf{1}_{4}^{\prime}-t \mathbf{V}^{(6)}, \\
& \mathbf{M}^{(7)}=2(t-2) \mathbf{1}_{4} \mathbf{1}_{4}^{\prime}-t\left(\begin{array}{llll}
0 & 3 & 1 & 2 \\
3 & 0 & 2 & 1 \\
1 & 2 & 0 & 3 \\
2 & 1 & 3 & 0
\end{array}\right)=2(t-2) \mathbf{1}_{4} \mathbf{1}_{4}^{\prime}-t \mathbf{V}^{(7)}
\end{aligned}
$$

or $\mathbf{M}^{(3)}$. Observe, that the case with two $3 \mathrm{~s}$ in one row is not excluded here, as more 3 s bring some additional 1 s. It can be calculated that $\lambda_{2}\left(\mathbf{V}^{(u)}\right) \geq 0$, 
$u=6,7$, and again from (8) $\lambda_{2}\left(\mathbf{S}_{d}+\mathbf{S}_{d}^{\prime}\right) \geq \lambda_{2}\left(\mathbf{V}^{(u)}\right)>0$ which satisfies condition (11) trivially. For $\mathbf{M}^{(3)}$ the thesis follows as in (b3).

(c) Let there exists at least one $i \neq j$ such that $\xi_{i j}=\xi \geq 4$. Then, we can find a submatrix of $\mathbf{G}_{d}$ of the form

$$
\mathbf{M}^{(8)}=2(t-2) \mathbf{1}_{2} \mathbf{1}_{2}^{\prime}-t\left(\begin{array}{cc}
0 & \xi \\
\xi & 0
\end{array}\right)
$$

for which $\lambda_{1}\left(\mathbf{M}^{(8)}\right)=t \xi>0$ and $\lambda_{2}\left(\mathbf{M}^{(1)}\right)=-8-t(\xi-4)<0$ for every $t$. From (7) $\lambda_{t}\left(\mathbf{G}_{d}\right) \leq \lambda_{2}\left(\mathbf{M}^{(8)}\right)<0$. Since additionally one of the eigenvalue of $\mathbf{G}_{d}$ is equal to zero, $\lambda_{t-1}\left(\mathbf{G}_{d}\right) \leq 0$ and (12) is satisfied trivially.

Step 3. Let $d \in \overline{\mathcal{R}}_{t, t-2, t} \backslash \mathcal{B}_{t, t-2, t}$. In the previous steps we have shown that for every $d_{b} \in \mathcal{B}_{t, t-2, t}$ inequality $\lambda_{t-1}\left(\mathbf{C}_{d^{*}}\right) \geq \lambda_{t-1}\left(\mathbf{C}_{d_{b}}\right)$ is satisfied. Observe, that if $d \in \overline{\mathcal{R}}_{t, t-2, t} \backslash \mathcal{B}_{t, t-2, t}$, there exists a design $d_{b}$ such that $\mathbf{S}_{d}=\mathbf{S}_{d_{b}}$. Moreover, from the optimality of BBD it is known that $\mathbf{N}_{d_{b}} \mathbf{N}_{d_{b}}^{\prime}=(t-2) \mathbf{1}_{t} \mathbf{1}_{t}^{\prime} \leq_{L} \mathbf{N}_{d} \mathbf{N}_{d}^{\prime}$, and thus $\mathbf{C}_{d} \leq_{L} \mathbf{C}_{d_{b}}$. Hence $\lambda_{t-1}\left(\mathbf{C}_{d}\right) \leq \lambda_{t-1}\left(\mathbf{C}_{d_{b}}\right) \leq \lambda_{t-1}\left(\mathbf{C}_{d^{*}}\right)$.

For $a<0$ there are some limitations for the class of optimality. Let $\mathcal{B}_{t, t-2, t}^{(3)}, t \geq 7$, be the class of binary designs for which:

(i) there exists at least one pair of unordered treatments that meet as the nearest neighbors three times,

(ii) every pair of unordered treatments appears as the nearest neighbors at most three times.

It means, that for every $d \in \mathcal{B}_{t, t-2, t}^{(3)}$ and $i \neq j, \xi_{i j}=s_{d, i j}+s_{d, j i} \in\{0,1,2,3\}$ and there exist at least one pair $(i, j)$ such that $\xi_{i j}=3$.

The following cases describe subclasses of $B_{t, t-2, t}^{(3)}$ :

- there exists exactly one pair $(i, j)$ such that $\xi_{i j}=3$ and at least one pair $\left(i^{\prime}, j^{\prime}\right)$ such that $\xi_{i^{\prime} j^{\prime}}=0$.

- there exists at least one pair $(i, j)$ such that $\xi_{i j}=3$ and at least one pair $\left(i^{\prime}, j^{\prime}\right)$ such that $\xi_{i^{\prime} j^{\prime}}=0$.

- for every $i \neq j, \xi_{i j} \neq 0$.

Let us denote by $\mathcal{B}_{t, t-2, t}^{(3,0)}$ the class of designs from $\mathcal{B}_{t, t-2, t}^{(3)}$ for which there is exactly one pair $(i, j)$ such that $\xi_{i j}=3$ and at least one pair $\left(i^{\prime}, j^{\prime}\right)$ such that $\xi_{i^{\prime} j^{\prime}}=0$. By $\mathcal{B}_{t, t-2, t}^{(3,1)}$ we denote $\mathcal{B}_{t, t-2, t}^{(3)} \backslash \mathcal{B}_{t, t-2, t}^{(3,0)}$.

Theorem 3 Let $t \geq 3$. If if there exists design $d^{*}$ with $\mathbf{S}_{d^{*}}$ permutationally similar to $\mathbf{1}_{t} \mathbf{1}_{t}^{\prime}-\mathbf{I}_{t}-\mathbf{H}_{t}$, then $d^{*}$ is E-optimal under model (1) with $A R(1, C)$ and $a<0$ over the class

(i) $\overline{\mathcal{R}}_{t, t-2, t}$ if $t \leq 6$,

(ii) $\overline{\mathcal{R}}_{t, t-2, t} \backslash \mathcal{B}_{t, t-2, t}^{(3,1)}$ if $t \geq 7$. 
Proof Let $a<0$. We prove the thesis in three steps.

Step 1. Let $d \in \widetilde{\mathcal{B}}_{t, t-2, t}$. We obtain the thesis by condition (10) and Lemma 2.

Step 2. Let $d \in \mathcal{B}_{t, t-2, t} \backslash \widetilde{\mathcal{B}}_{t, t-2, t}$. We have to show that

$$
\lambda_{t}\left(\mathbf{S}_{d^{*}}+\mathbf{S}_{d^{*}}^{\prime}\right) \geq \lambda_{t}\left(\mathbf{S}_{d}+\mathbf{S}_{d}^{\prime}\right)
$$

Note that $\lambda_{t}\left(\mathbf{S}_{d^{*}}+\mathbf{S}_{d^{*}}^{\prime}\right) \geq-4$. Since the diagonal entries of $\xi_{i i}=0$ and $\xi_{i j} \in$ $\{0,1, \ldots, t-2\}$, we will consider several cases of $\mathbf{S}_{d}+\mathbf{S}_{d}^{\prime}$.

(a) If the off-diagonal entries $\xi_{i j} \leq 2$ for every $i \neq j$, then there exists a design $\widetilde{d} \in \widetilde{\mathcal{B}}_{t, t-2, t}$ such that $\mathbf{S}_{d}+\mathbf{S}_{d}^{\prime}=\mathbf{S}_{\widetilde{d}}+\mathbf{S}_{\widetilde{d}}^{\prime}$ and the thesis follows from Lemma 2.

(b) Assume that $t \leq 6$ and $\xi_{i j} \in\{0,1,2,3\}$ for every $i \neq j$ and there exists at least one $i \neq j$ such that $\xi_{i j}=3$. Then we can find a submatrix of $\mathbf{S}_{d}+\mathbf{S}_{d}^{\prime}$ of the form

$$
\mathbf{M}^{(1)}=\left(\begin{array}{ll}
0 & 3 \\
3 & 0
\end{array}\right)
$$

for which $\lambda_{2}\left(\mathbf{M}^{(1)}\right)=-3$. From (8) we have $\lambda_{t}\left(\mathbf{S}_{d}+\mathbf{S}_{d}^{\prime}\right) \leq \lambda_{2}\left(\mathbf{M}^{(1)}\right)=-3$ and since $\lambda_{t}\left(\mathbf{S}_{d^{*}}+\mathbf{S}_{d^{*}}^{\prime}\right) \geq-3$ for $t \leq 6$ we obtain (13).

(c) Let $t \geq 7$ and let $d \in \mathcal{B}_{t, t-2, t}^{(3,1)}$.

Assume that there is exactly one $\xi_{d, i^{\prime} j^{\prime}}=0$, which is in the same row as 3, i.e., $i^{\prime}=i$. Because of the fixed sums of rows and columns of $\mathbf{S}_{d}$, it can be observed that:

- $(t-4)$ off-diagonal entries of $\mathbf{S}_{d}+\mathbf{S}_{d}^{\prime}$ must be equal to 2 , one entry is equal to 0 , one is equal to 1 and one is equal to 3 in the $i$ th row;

- $(t-5)$ off-diagonal entries of $\mathbf{S}_{d}+\mathbf{S}_{d}^{\prime}$ must be equal to 2 , three are equal to 1 and one is equal to 3 in the $j$ th row;

- $(t-2)$ off-diagonal entries of $\mathbf{S}_{d}+\mathbf{S}_{d}^{\prime}$ must be equal to 2 , and one is equal to 0 in the row $j^{\prime}$;

- $(t-3)$ off-diagonal entries of $\mathbf{S}_{d}+\mathbf{S}_{d}^{\prime}$ must be equal to 2 and two are equal to 1 in the remaining rows.

Thus for every $\mathbf{S}_{d}+\mathbf{S}_{d}^{\prime}$ there exists a submatrix

$$
\mathbf{M}^{(2)}=\left(\begin{array}{llll}
0 & 3 & 0 & 2 \\
3 & 0 & 2 & 1 \\
0 & 2 & 0 & 2 \\
2 & 1 & 2 & 0
\end{array}\right)
$$

for which $\lambda_{4}\left(\mathbf{M}^{(2)}\right)=-4.11$. From (8) $\lambda_{t}\left(\mathbf{S}_{d}+\mathbf{S}_{d}^{\prime}\right) \leq \lambda_{4}\left(\mathbf{M}^{(2)}\right)<-4 \leq$ $\lambda_{t}\left(\mathbf{S}_{d^{*}}+\mathbf{S}_{d^{*}}^{\prime}\right)$. 
Assume now that there is exactly one $\xi_{d, i^{\prime} j^{\prime}}=0$, which is in different row than 3 , i.e., $i^{\prime} \neq i$. Then, we can find a submatrix of $\mathbf{S}_{d}+\mathbf{S}_{d}^{\prime}$ permutationally similar to

$$
\mathbf{M}^{(3)}=\left(\begin{array}{llll}
0 & 0 & 2 & 2 \\
0 & 0 & 2 & 2 \\
2 & 2 & 0 & 3 \\
2 & 2 & 3 & 0
\end{array}\right) \text {. }
$$

Moreover, in the row $i^{\prime}$ the only possible off-diagonal entries different than 0 are 2s. Thus, one of the eigenvectors of $\mathbf{S}_{d}+\mathbf{S}_{d}^{\prime}$ is of the form $\left(\alpha_{1} \mathbf{1}_{2}^{\prime}: \alpha_{2} \mathbf{1}_{t-2}^{\prime}\right)^{\prime}$ and it corresponds to the -4 egienvalue of $\mathbf{S}_{d}+\mathbf{S}_{d}^{\prime}$. From (8) we obtain the thesis.

Let now assume that there exist at least two pairs of indices $\left(i^{\prime}, j^{\prime}\right),\left(i^{\prime \prime}, j^{\prime \prime}\right)$ such that $\xi_{i^{\prime} j^{\prime}}=\xi_{i^{\prime \prime} j^{\prime \prime}}=0$. Observe that the only possible situation is $i^{\prime} \neq i^{\prime \prime}$ and $j^{\prime} \neq j^{\prime \prime}$. Thus, there are one off-diagonal 0 in rows $j^{\prime}, j^{\prime \prime}$ with the remaining elements off-diagonal entries are equal to 2. Hence, there exist a submatrix of $\mathbf{S}_{d}+\mathbf{S}_{d}^{\prime}$ of the form

$$
\mathbf{M}^{(4)}=\left(\begin{array}{llll}
0 & 3 & 0 & 2 \\
3 & 0 & 2 & 0 \\
0 & 2 & 0 & 2 \\
2 & 0 & 2 & 0
\end{array}\right)
$$

for which $\lambda_{4}\left(\mathbf{M}^{(4)}\right)=-4$. From (8) we obtain the thesis.

(d) Let there exist at least one $i, j$ such that $\xi_{i j} \geq 4$. Then there exists a submatrix

$$
\mathbf{M}^{(2)}=\left(\begin{array}{cc}
0 & \xi_{i j} \\
\xi_{i j} & 0
\end{array}\right)
$$

for which $\lambda_{2}\left(\mathbf{M}^{(3)}\right)=-\xi_{i j} \leq-4$. By $(8), \lambda_{t}\left(\mathbf{S}_{d}+\mathbf{S}_{d}^{\prime}\right) \leq \lambda_{2}\left(\mathbf{M}^{(2)}\right) \leq-4$ and (13) is satisfied.

Step 3. If $d \in \overline{\mathcal{R}}_{t, t-2, t} \backslash \mathcal{B}_{t, t-2, t}$ then the proof follows the same lines as in Theorem 2.

It is worth noting that $\mathcal{B}_{t, t-2, t}^{(3,1)}$ is a class which can contain better design than $d^{*}$. For example, if $t=7$ then there exists design

$$
d^{\#}=\left(\begin{array}{ccccccc}
1 & 2 & 3 & 4 & 5 & 6 & 7 \\
1 & 2 & 4 & 3 & 7 & 5 & 6 \\
1 & 3 & 5 & 7 & 2 & 6 & 4 \\
1 & 3 & 6 & 7 & 5 & 2 & 4 \\
1 & 3 & 2 & 4 & 7 & 6 & 5
\end{array}\right)
$$


with blocks represented as rows, such that

$$
\mathbf{S}_{d^{\#}}+\mathbf{S}_{d^{\#}}^{\prime}=\left(\begin{array}{ccccccc}
0 & 2 & 3 & 2 & 1 & 1 & 1 \\
2 & 0 & 2 & 3 & 1 & 1 & 1 \\
3 & 2 & 0 & 2 & 1 & 1 & 1 \\
2 & 3 & 2 & 0 & 1 & 1 & 1 \\
1 & 1 & 1 & 1 & 0 & 3 & 3 \\
1 & 1 & 1 & 1 & 3 & 0 & 3 \\
1 & 1 & 1 & 1 & 3 & 3 & 0
\end{array}\right)
$$

and $\lambda_{7}\left(\mathbf{S}_{d^{\#}}+\mathbf{S}_{d^{\#}}^{\prime}\right)=-3$. Observe, that in this case $\lambda_{7}\left(\mathbf{S}_{d^{*}}+\mathbf{S}_{d^{*}}^{\prime}\right)=-2+$ $2 \cos (6 \pi / 7) \approx-3.8$ and thus $\lambda_{6}\left(\mathbf{C}_{d^{\#}}\right)>\lambda_{6}\left(\mathbf{C}_{d^{*}}\right)$. Moreover, $d^{\#}$ is E-optimal over the class of all $\overline{\mathcal{R}}_{7,5,7}$ because

- the highest $\lambda_{7}\left(\mathbf{S}_{d}+\mathbf{S}_{d}^{\prime}\right)$ over $d \in \widetilde{\mathcal{B}}_{7,5,7}$ is -3.8 ;

- if there exist at least one $i, j$, such that $\xi_{i j} \geq 3$ then we can find a submatrix

$$
\mathbf{M}_{d}=\left(\begin{array}{cc}
0 & \xi_{i j} \\
\xi_{j i} & 0
\end{array}\right)
$$

for which $\lambda_{2}\left(\mathbf{M}_{d}\right)=-\xi_{i j} \leq-3$;

- optimality over the class $\overline{\mathcal{R}}_{7,5,7}$ follows directly from Theorem 3 .

For $t \geq 8$, if $d \in \mathcal{B}_{t, t-2, t}^{(3,1)}$ there are a lot of possible forms of $\mathbf{S}_{d}+\mathbf{S}_{d}^{\prime}$. We are not aware of finding any general method for proving E-optimality of $d^{*}$ over $\mathcal{B}_{t, t-2, t}^{(3,1)}$ however we conjecture that $d^{*}$ is E-optimal over at least $\overline{\mathcal{R}}_{t, t-2, t}$.

Example 1 For $t=3,5,7$, the designs satisfying conditions of Theorems 2 and 3 can be constructed from CNBDs by removing one block, since in these cases $\mathbf{S}_{d^{*}}$ are permutationally similar to $\mathbf{1}_{t} \mathbf{1}_{t}^{\prime}-\mathbf{I}_{t}-\mathbf{H}_{t}$. The E-optimal designs are respectively

$$
d^{*}=\left(\begin{array}{lll}
1 & 2 & 3
\end{array}\right), \quad d^{*}=\left(\begin{array}{lllll}
1 & 2 & 3 & 4 & 5 \\
1 & 3 & 5 & 2 & 4 \\
1 & 4 & 2 & 5 & 3
\end{array}\right), \quad d^{*}=\left(\begin{array}{lllllll}
1 & 2 & 3 & 4 & 5 & 6 & 7 \\
1 & 3 & 5 & 7 & 2 & 4 & 6 \\
1 & 4 & 7 & 3 & 6 & 2 & 5 \\
1 & 5 & 2 & 6 & 3 & 7 & 4 \\
1 & 6 & 4 & 2 & 7 & 5 & 3
\end{array}\right)
$$

with blocks represented by rows.

It is worth noting that E-optimal designs presented in Theorem 3 are also E-optimal under the one-sided interference model with uncorrelated observations (cf. Filipiak et al. 2008) and thus, some of the construction methods of E-optimal designs can be adopted. 


\subsection{E-optimal complete block designs for $b=t$}

Let define the following subclass of $\mathcal{B}_{t, t, t}$ :

$$
\widehat{\mathcal{B}}_{t, t, t}=\left\{d: \quad \mathbf{S}_{d}=\mathbf{1}_{t} \mathbf{1}_{t}^{\prime}-\mathbf{I}_{t}+\mathbf{P}_{d}, \mathbf{P}_{d} \in \overline{\mathbf{P}}_{t}\right\}
$$

For a design $d \in \widehat{\mathcal{B}}_{t, t, t}$,

$$
\mathbf{C}_{d}=\left(t\left(1+a^{2}\right)+2 a\right) \mathbf{I}_{t}-\left(1+a^{2}\right) \mathbf{1}_{t} \mathbf{1}_{t}^{\prime}-a\left(\mathbf{P}_{d}+\mathbf{P}_{d}^{\prime}\right)
$$

Similarly to the previous section E-optimality condition can be expressed as

$$
\begin{gathered}
\lambda_{2}\left(\mathbf{P}_{d^{*}}+\mathbf{P}_{d^{*}}^{\prime}\right) \leq \lambda_{2}\left(\mathbf{P}_{d}+\mathbf{P}_{d}^{\prime}\right) \text { if } a>0 \\
\lambda_{t}\left(\mathbf{P}_{d^{*}}+\mathbf{P}_{d^{*}}^{\prime}\right) \geq \lambda_{t}\left(\mathbf{P}_{d}+\mathbf{P}_{d}^{\prime}\right) \text { if } a<0 .
\end{gathered}
$$

Inequalities (14) and (15) correspond respectively to inequalities (10) and (9) from Sect. 4.1.

For simplicity of the proofs of the next theorems, for every $d \in \mathcal{B}_{t, t, t}$ let define $\mathbf{Q}_{d}=\left(q_{d, i j}\right)$ as

$$
\mathbf{Q}_{d}=2 \mathbf{1}_{t} \mathbf{1}_{t}^{\prime}-\left(\mathbf{S}_{d}+\mathbf{S}_{d}^{\prime}\right) .
$$

It is clear that all diagonal entries of $\mathbf{Q}_{d}$ are equal to 2 and for every $i \neq j$

$$
q_{d, i j} \in\{2,1, \ldots, 2-2 t\} .
$$

Moreover, $\mathbf{Q}_{d} \mathbf{1}_{t}=0 \cdot \mathbf{1}_{t}$. Since the elements of $\mathbf{S}_{d}+\mathbf{S}_{d}^{\prime}$ are denoted by $\xi_{i j}$ in Sect. 2, it is clear that $q_{d, i j}=2-\xi_{i j}$.

If $a>0$ there are some limitations for the class of optimality. For $t \geq 7$ let $\mathcal{B}_{t, t, t}^{(1)}$ be the class of binary designs such that

(i) there exists at least one pair of unordered treatments that meet as the nearest neighbors once,

(ii) every pair of unordered treatments appears as the nearest neighbors at least once and at most three times.

It means, that for every $d \in \mathcal{B}_{t, t, t}^{(1)}$ and $i \neq j, \xi_{i j}=s_{d, i j}+s_{d, j i} \in\{1,2,3\}$ and there exists at least one pair $(i, j)$ such that $\xi_{i j}=1$.

Theorem 4 If there exists design $d^{*}$ with $\mathbf{S}_{d^{*}}$ permutationally similar to $\mathbf{1}_{t} \mathbf{1}_{t}^{\prime}-\mathbf{I}_{t}+\mathbf{H}_{t}$, then $d^{*}$ is E-optimal under model (1) with $A R(1, C)$ and $a>0$ over the class

(i) $\overline{\mathcal{R}}_{t, t, t}$ if $t \leq 6$,

(ii) $\overline{\mathcal{R}}_{t, t, t} \backslash \mathcal{B}_{t, t, t}^{(1)}$ if $t \geq 7$.

Proof Let $a>0$. We prove the thesis in three steps.

Step 1. Let $d \in \widehat{\mathcal{B}}_{t, t, t}$. The thesis follows from (14) and Lemma 2.

Step 2. Let $d \in \mathcal{B}_{t, t, t} \backslash \widehat{\mathcal{B}}_{t, t, t}$. To show (11) it is enough to prove

$$
\lambda_{t-1}\left(\mathbf{Q}_{d}\right) \leq \lambda_{t-1}\left(\mathbf{Q}_{d^{*}}\right)
$$


Note that $\lambda_{t-1}\left(\mathbf{Q}_{d^{*}}\right) \geq 0$. We consider several cases of $\mathbf{Q}_{d}$.

(a) Let $\xi_{i j} \in\{2,3,4\}$ for every $i \neq j$. Thus, there exists $\hat{d} \in \widehat{\mathcal{B}}_{t, t, t}$ such that $\mathbf{S}_{d}+\mathbf{S}_{d}^{\prime}=$ $\mathbf{S}_{\hat{d}}+\mathbf{S}_{\hat{d}}^{\prime}$ and the thesis follows from Lemma 2 .

(b) Let $\xi_{i j} \in\{0,1,2,3,4\}$ for every $i \neq j$. Assume that there exists at least one pair $(i, j)$ such that $\xi_{i j}=0$. Then there exists a $2 \times 2$ zero submatrix of $\mathbf{S}_{d}+\mathbf{S}_{d}^{\prime}$, for which trivially the eigenvalues are equal to 0 . From (8) we obtain the thesis.

(c) Assume now that $\xi_{i j} \in\{1,2,3,4\}$ for every $i \neq j$ and there exists at least one pair $\left(i^{\prime}, j^{\prime}\right)$ such that $\xi_{i^{\prime} j^{\prime}}=1$. We cosider two cases separetely.

(c1) Let $t \leq 6$ and let $\xi_{i j} \in\{1,2,3\}$ for every $i, j=1, \ldots, t$. Since the rows and columns of $\mathbf{Q}_{d}$ sum to zero and the diagonal entries are equal to 2 , there exists a submatrix of $\mathbf{Q}_{d}$ of the form

$$
\mathbf{M}^{(1)}=2 \mathbf{1}_{3} \mathbf{1}_{3}^{\prime}-\left(\begin{array}{lll}
0 & 1 & 3 \\
1 & 0 & 3 \\
3 & 3 & 0
\end{array}\right) .
$$

From (7) we have $\lambda_{t-1}\left(\mathbf{Q}_{d}\right) \leq \lambda_{2}\left(\mathbf{M}^{(1)}\right)=1 \leq 2\left(1-\cos \left(\frac{2 \pi}{t}\right)\right)=$ $\lambda_{t-1}\left(\mathbf{Q}_{d^{*}}\right)$.

(c2) Let $t \geq 3$ and $d \in \mathcal{B}_{t, t, t} \backslash \mathcal{B}_{t, t, t}^{(1)}$. It means that $\xi_{i j} \in\{1,2,3,4\}$ and there exists at least one pair $(i, j)$ such that $\xi_{i j}=4$. Then we can find a submatrix of $\mathbf{Q}_{d}$ of the form

$$
\mathbf{M}^{(2)}=2 \mathbf{1}_{4} \mathbf{1}_{4}^{\prime}-\left(\begin{array}{cccc}
0 & 1 & 4 & \xi_{1} \\
1 & 0 & \xi_{2} & \xi_{3} \\
4 & \xi_{2} & 0 & \xi_{4} \\
\xi_{1} & \xi_{3} & \xi_{4} & 0
\end{array}\right)
$$

with $\xi_{m} \in\{1,2,3,4\}, m=1,2,3,4$. It is easy to verify that $\operatorname{det}\left(\mathbf{M}^{(2)}\right)<0$ for every $\xi_{m}$. It implies that there exists at least one negative eigenvalue of $\mathbf{M}^{(2)}$ (and from (7) also of $\mathbf{Q}_{d}$ ). Since one of the eigenvalues of $\mathbf{Q}_{d}$ is zero we obtain that $\lambda_{t-1}\left(\mathbf{Q}_{d}\right) \leq 0 \leq \lambda_{t-1}\left(\mathbf{Q}_{d}^{*}\right)$.

(d) Let there exist at least one pair $(i, j)$ such that $\xi_{i j} \geq 5$. Then there exists a submatrix

$$
\mathbf{M}^{(3)}=2 \mathbf{1}_{2} \mathbf{1}_{2}^{\prime}-\left(\begin{array}{cc}
0 & \xi_{i j} \\
\xi_{j i} & 0
\end{array}\right) .
$$

Since $\operatorname{det}\left(\mathbf{M}^{(3)}\right)<0$ the thesis is proved by the same lines as in (c2).

Step 3. If $d \in \overline{\mathcal{R}}_{t, t, t} \backslash \mathcal{B}_{t, t, t}$ then the proof follows the same lines as in Theorem 2. 
It is worth noting that $\mathcal{B}_{t, t, t}^{(1)}$ is a class which can contain better design than $d^{*}$. For example, if $t=7$ then there exists design (with blocks represented as rows)

$$
d^{\#}=\left(\begin{array}{lllllll}
1 & 2 & 3 & 4 & 5 & 6 & 7 \\
1 & 5 & 2 & 6 & 3 & 7 & 4 \\
1 & 6 & 4 & 7 & 2 & 5 & 3 \\
1 & 7 & 3 & 5 & 2 & 4 & 6 \\
1 & 5 & 4 & 6 & 3 & 2 & 7 \\
1 & 4 & 3 & 5 & 7 & 2 & 6 \\
1 & 2 & 6 & 3 & 7 & 4 & 5
\end{array}\right) \text {, with } \mathbf{S}_{d^{\#}}+\mathbf{S}_{d^{\#}}^{\prime}=\left(\begin{array}{ccccccc}
0 & 2 & 1 & 2 & 3 & 3 & 3 \\
2 & 0 & 2 & 1 & 3 & 3 & 3 \\
1 & 2 & 0 & 2 & 3 & 3 & 3 \\
2 & 1 & 2 & 0 & 3 & 3 & 3 \\
3 & 3 & 3 & 3 & 0 & 1 & 1 \\
3 & 3 & 3 & 3 & 1 & 0 & 1 \\
3 & 3 & 3 & 3 & 1 & 1 & 0
\end{array}\right)
$$

and $\lambda_{2}\left(\mathbf{S}_{d^{\#}}+\mathbf{S}_{d^{\#}}^{\prime}\right)=-1<\lambda_{2}\left(\mathbf{S}_{d^{*}}+\mathbf{S}_{d^{*}}^{\prime}\right)=-0.75$. Thus $\lambda_{6}\left(\mathbf{C}_{d^{\#}}\right)>\lambda_{6}\left(\mathbf{C}_{d^{*}}\right)$. Moreover, $d^{\#}$ is E-optimal over the class of all $\overline{\mathcal{R}}_{7,7,7}$ because

- the smallest $\lambda_{2}\left(\mathbf{S}_{d}+\mathbf{S}_{d}^{\prime}\right)$ over $d \in \widehat{\mathcal{B}}_{7,5,7}$ is -0.75 ;

- if $\xi_{i j} \in\{1,2,3\}$ and there exists at least one pair $(i, j)$, such that $\xi_{i j}=1$. Then we can find submatrix $\mathbf{1}_{2} \mathbf{1}_{2}^{\prime}-\mathbf{I}_{2}$ of $\mathbf{S}_{d}+\mathbf{S}_{d}^{\prime}$ for which $\lambda_{2}\left(\mathbf{1}_{2} \mathbf{1}_{2}^{\prime}-\mathbf{I}_{2}\right)=-1$. By (8) we obtain $\lambda_{2}\left(\mathbf{S}_{d}+\mathbf{S}_{d}^{\prime}\right) \geq-1=\lambda_{2}\left(\mathbf{S}_{d^{\#}}+\mathbf{S}_{d^{\#}}^{\prime}\right)$ and finally $\lambda_{6}\left(\mathbf{C}_{d}\right) \leq \lambda_{6}\left(\mathbf{C}_{d^{\#}}\right)$; - optimality over the class $\overline{\mathcal{R}}_{7,7,7}$ follows directly from Theorem 4 .

For $t \geq 8$, if $d \in \mathcal{B}_{t, t, t}^{(1)}$ there are a lot of possible forms of $\mathbf{S}_{d}+\mathbf{S}_{d}^{\prime}$. We are not aware of finding any general method for proving E-optimality of $d^{*}$ over $\mathcal{B}_{t, t, t}^{(1)}$ however we conjecture that $d^{*}$ is E-optimal over at least $\overline{\mathcal{R}}_{t, t, t}$.

Theorem 5 If there exists design $d^{*}$ with $\mathbf{S}_{d^{*}}=\mathbf{1}_{t} \mathbf{1}_{t}^{\prime}-\mathbf{I}_{t}+\mathbf{P}_{d^{*}}$, such that $\mathbf{P}_{d^{*}}$ is permutationally similar to the matrix

(i) $\mathbf{H}_{t}$ if $t=2,7$;

(ii) $\mathbf{I}_{2} \otimes \mathbf{H}_{2}$ or $\mathbf{H}_{4}$ if $t=4$;

(iii) $\mathbf{I}_{m} \otimes \mathbf{H}_{3}$ if $t=3 m, m \in \mathbb{N}$;

(iv) $\operatorname{diag}\left(\mathbf{I}_{i} \otimes \mathbf{H}_{3}, \mathbf{I}_{j} \otimes \mathbf{H}_{5}\right)$ if $t=5$ or $t \geq 8$ and $t \neq 3 m, m \in \mathbb{N}$ with $t=3 i+5 j$ for some $i \in \mathbb{N} \cup\{0\}$ and $j \in \mathbb{N}$;

then $d^{*}$ is E-optimal under model 1 with $A R(1, C)$ and $a<0$ over the class $\overline{\mathcal{R}}_{t, t, t}$.

Proof Let $a<0$. We prove the thesis in three steps.

Step 1. Let $d \in \widehat{\mathcal{B}}_{t, t, t}$. The thesis follows from (15) and Lemma 1

Step 2. Let $d \in \mathcal{B}_{t, t, t} \backslash \widehat{\mathcal{B}}_{t, t, t}$. To show (13) it is enough to prove

$$
\lambda_{1}\left(\mathbf{Q}_{d^{*}}\right) \leq \lambda_{1}\left(\mathbf{Q}_{d}\right)
$$

Note that $\lambda_{1}\left(\mathbf{Q}_{d^{*}}\right) \leq 4$. We consider several cases of $\mathbf{Q}_{d}$.

(a) Let $\xi_{i j} \in\{2,3\}$ for every $i \neq j$. Thus, there exists $\hat{d} \in \widehat{\mathcal{B}}_{t, t, t}$ such that $\mathbf{S}_{d}+\mathbf{S}_{d}^{\prime}=$ $\mathbf{S}_{\hat{d}}+\mathbf{S}_{\hat{d}}^{\prime}$ and the thesis follows from Lemma 1.

(b) Let there exists at least one pair $i \neq j$ such that $\xi_{i j} \in\{0,4,5, \ldots, t\}$. Then we can find a submatrix

$$
\mathbf{M}^{(1)}=2 \mathbf{1}_{2} \mathbf{1}_{2}^{\prime}-\left(\begin{array}{cc}
0 & \xi_{i j} \\
\xi_{i j} & 0
\end{array}\right)
$$


for which $\lambda_{1}\left(\mathbf{M}^{(1)}\right) \geq 4$. By (8) we have $\lambda_{1}\left(\mathbf{Q}_{d}\right) \geq \lambda_{1}\left(\mathbf{M}^{(1)}\right) \geq 4 \geq \lambda_{1}\left(\mathbf{Q}_{d^{*}}\right)$.

(c) Assume now that $\xi_{i j} \in\{1,2,3\}$ for every $i \neq j$ and there exists at least one pair $(i, j)$ such that $\xi_{i j}=1$. Because of the fixed sums of rows and columns of $\mathbf{S}_{d}$, it can be observed that:

- if there exists exactly one 1 in a row of $\mathbf{S}_{d}+\mathbf{S}_{d}^{\prime}$ then there are $(t-5)$ off-diagonal entries of $\mathbf{S}_{d}+\mathbf{S}_{d}^{\prime}$ equal to 2 and three entries equal to 3 in this row;

- if there exist more than one 1 in a row of $\mathbf{S}_{d}+\mathbf{S}_{d}^{\prime}$ then there are more than three entries equal to 3 in this row.

Due to these observations we consider two cases.

(c1) If there exists exactly one pair of $i \neq j$ such that $\xi_{i j}=1$, then we find one of the following submatrices of $\mathbf{Q}_{d}$ :

$$
\begin{gathered}
\mathbf{M}^{(2)}=2 \mathbf{1}_{3} \mathbf{1}_{3}^{\prime}-\left(\begin{array}{lll}
0 & 1 & 3 \\
1 & 0 & 3 \\
3 & 3 & 0
\end{array}\right), \\
\mathbf{M}^{(3)}=2 \mathbf{1}_{5} \mathbf{1}_{5}^{\prime}-\left(\begin{array}{lllll}
0 & 1 & 2 & 2 & 3 \\
1 & 0 & 3 & 2 & 2 \\
2 & 3 & 0 & 3 & 2 \\
2 & 2 & 3 & 0 & 3 \\
3 & 2 & 2 & 3 & 0
\end{array}\right), \\
\mathbf{M}^{(4)}=2 \mathbf{1}_{8} \mathbf{1}_{8}^{\prime}-\left(\begin{array}{llllllll}
0 & 1 & 3 & 3 & 3 & 2 & 2 & 2 \\
1 & 0 & 2 & 2 & 2 & 3 & 3 & 3 \\
3 & 2 & 0 & 3 & 2 & 2 & 2 & 2 \\
3 & 2 & 3 & 0 & 2 & 2 & 2 & 2 \\
3 & 2 & 2 & 2 & 0 & 3 & 2 & 2 \\
2 & 3 & 2 & 2 & 3 & 0 & 2 & 2 \\
2 & 3 & 2 & 2 & 2 & 2 & 0 & 3 \\
2 & 3 & 2 & 2 & 2 & 2 & 3 & 0
\end{array}\right) .
\end{gathered}
$$

It can be calculated that $\lambda_{1}\left(\mathbf{M}^{(m)}\right)=4$ for $m=2,3,4$. Thus by (8) inequality (16) is satisfied.

(c2) If there exists more than one pair of $i \neq j$ such that $\xi_{i j}=1$, then we can find one of the submatrices of (c1) or:

$$
\begin{aligned}
& \mathbf{M}^{(5)}=2 \mathbf{1}_{4} \mathbf{1}_{4}^{\prime}-\left(\begin{array}{llll}
0 & 1 & 3 & 2 \\
1 & 0 & 2 & 3 \\
3 & 2 & 0 & 1 \\
2 & 3 & 1 & 0
\end{array}\right), \\
& \mathbf{M}^{(6)}=2 \mathbf{1}_{4} \mathbf{1}_{4}^{\prime}-\left(\begin{array}{llll}
0 & 1 & 3 & 3 \\
1 & 0 & 2 & 2 \\
3 & 2 & 0 & 1 \\
3 & 2 & 1 & 0
\end{array}\right),
\end{aligned}
$$




$$
\mathbf{M}^{(7)}=2 \mathbf{1}_{6} \mathbf{1}_{6}^{\prime}-\left(\begin{array}{cccccc}
0 & 1 & 3 & 3 & 2 & 2 \\
1 & 0 & 2 & 2 & 3 & 3 \\
3 & 2 & 0 & 2 & 2 & 2 \\
3 & 2 & 2 & 0 & 2 & 2 \\
2 & 3 & 2 & 2 & 0 & 2 \\
2 & 3 & 2 & 2 & 2 & 0
\end{array}\right)
$$

It can be calculated that $\lambda_{1}\left(\mathbf{M}^{(m)}\right) \geq 4$ for $m=5,6,7$. Thus by (8) inequality (16) is satisfied.

It is worth noting that E-optimal designs presented in Theorem 5 are also E-optimal under the one-sided interference model with uncorrelated observations [cf. Filipiak et al. (2008)] and thus, some of the construction methods of E-optimal designs can be adopted from Filipiak et al. (2008) or Filipiak and Różański (2005). Moreover, for $a>0$ E-optimal designs can be constructed from CNBDs by repeating one block.

Example 2 For $t=6$, the designs satisfying conditions of Theorem 4 is $d_{1}^{*}$, whilst for arbitrary $a$ and $t=4$ an example of E-optimal design is $d_{2}^{*}$, with

$$
d_{1}^{*}=\left(\begin{array}{cccccc}
1 & 2 & 3 & 4 & 5 & 6 \\
1 & 2 & 4 & 6 & 5 & 3 \\
1 & 3 & 2 & 5 & 6 & 4 \\
1 & 4 & 5 & 2 & 6 & 3 \\
1 & 5 & 4 & 3 & 6 & 2 \\
1 & 6 & 4 & 2 & 3 & 5
\end{array}\right), \quad d_{2}^{*}=\left(\begin{array}{llll}
1 & 4 & 3 & 2 \\
1 & 2 & 3 & 4 \\
1 & 2 & 4 & 3 \\
1 & 3 & 4 & 2
\end{array}\right)
$$

and blocks represented by rows.

\section{Concluding remarks}

In the paper universal optimality of some circular NdNBD designs over the class of circular designs with arbitrary block size under $\mathrm{AR}(1, C)$ and $a>0$ is shown. For $a<0$ universal optimality of these designs over the class of circular designs with no treatment preceded by itself is proven. The only limitation on design parameters in this case is the existence of BBD design with completely symmetric nondirected neighboring matrix.

If a circular universally optimal BBD cannot exist, E-optimality is considered in the class of complete block designs. If the number of blocks is smaller by two than the number of treatments and the correlation parameter $a$ is positive as well as if the number of blocks is equal to the number of treatments and the correlation parameter is negative, the left-neighboring matrix of an E-optimal design over the class of all equireplicated designs without self-neighbors is given. In the remaining cases the class of optimality is more limited, however, we conjecture that the designs with leftneighboring matrices presented in Theorems 3 and 4 are still optimal over at least the class of equireplicated designs with no treatment preceded by itself. 
In all the cases at least one example of E-optimal design is given. To construct such designs one can regard the left-neighboring matrix as an adjacency matrix of a directed graph and use its decomposition into Hamiltonian cycles. Such a decomposition can be done for example with the use of FINDHAMILTONIANCYCLES procedure available in Mathematica 9.0 and later versions. The problem however is that looking for such a decomposition is extremely time-consuming even for relatively small number of treatments (vertices in graphs). Therefore some combinatorial methods of construction of E-optimal designs will be subject of the future research. It is especially interesting because optimal designs in the standard block effects model with observations correlated with respect to circular autoregression can be also shown to be optimal in more general models, e.g. with carry-over effects as additional nuisance parameters.

Acknowledgements This research is partially supported by Statutory Activities (K. Filipiak).

Open Access This article is distributed under the terms of the Creative Commons Attribution 4.0 International License (http://creativecommons.org/licenses/by/4.0/), which permits unrestricted use, distribution, and reproduction in any medium, provided you give appropriate credit to the original author(s) and the source, provide a link to the Creative Commons license, and indicate if changes were made.

\section{References}

Azaïs JM, Bailey RA, Monod H (1993) A catalogue of efficient neighbour-designs with border plots. Biometrics 49:1252-1261

Bailey RA, Cameron PJ, Filipiak K, Kunert J, Markiewicz A (2017) On optimality and construction of circular repeated-measurements designs. Stat Sin 27:1-22

Bayer MM, Todd CD (1996) Effect of polypide regression and other parameters on colony growth in the cheilostomate Electra pilosa (L.). In: Gordon DP, Smith AM, Grant-Mackie JA (eds) Bryozoans in space and time. National Institute of Water and Atmospheric Research, Wellington, NZ, pp 29-38

Chai FS, Majumdar D (2000) Optimal designs for nearest-neighbor analysis. J Stat Plan Inference 86:265275

Das A, Dey A (1989) A note on balanced block designs. J Stat Plan Inference 22:256-268

Druilhet P (1999) Optimality of circular neighbour balanced designs. J Stat Plan Inference 81:141-152

Filipiak K, Różański R, Sawikowska A, Wojtera-Tyrakowska D (2008) On the E-optimality of complete designs under an interference model. Stat Probab Lett 78:2470-2477

Filipiak K, Markiewicz A (2003) Optimality of circular neighbor balanced designs under mixed effects model. Stat Probab Lett 61:225-234

Filipiak K, Markiewicz A (2004) Optimality of type I orthogonal arrays for general interference model with correlated observations. Stat Probab Lett 68:259-265

Filipiak K, Markiewicz A (2005) Optimality and efficiency of circular neighbor balanced designs for correlated observations. Metrika 61:17-27

Filipiak K, Markiewicz A (2007) Optimal designs for a mixed interference model. Metrika 65:369-386

Filipiak K, Różański R (2005) E-optimal designs under an interference model. Biom Lett 42(2):133-142

Gill PS, Shukla GK (1985) Efficiency of nearest neighbour balanced block designs for correlated observations. Biometrika 72:539-544

Jacroux M (1982) Some E-optimal designs for the one-way and two-way elimination of heterogeneity. J R Stat Soc B 44:253-261

Jacroux M (1983) On the E-optimality of block designs. Sankhya Ser B 45:351-361

Kiefer J (1958) On the nonrandomized optimality and randomized nonoptimality of symmetrical designs. Ann Math Stat 29:675-699

Kiefer J (1975) Construction and optimality of generalised Youden designs. In: Srivastava JN (ed) A survey of statistical design and linear models. North Holland, Amsterdam, pp 333-353

Kiefer J, Wynn H (1981) Optimum balanced block and latin square designs for correlated observations. Ann Stat 9:737-757 
Khodsiani R, Pooladsaz S (2017) Universal optimal block designs under hub correlation structure. Stat Probab Lett 129:387-392

Kunert J (1987) Neighbour balanced block designs for correlated errors. Biometrika 74:717-724

Kunert J, Martin R (1987) On the optimality of finite Williams type II designs. Ann Stat 15:1604-1628

Kunert J, Martin R (1987) Some results on optimal design under a first-order autoregression and on finite Williams type II designs. Commun Stat Theory Methods 16:1901-1922

Marshall AW, Olkin I, Arnold BC (2011) Inequalities: theory of majorization and its applications, 2nd edn. Springer, Berlin

Pooladsaz S, Martin RJ (2005) Optimal extended complete block designs for dependent observations. Metrika 61:185-197

Pukelsheim F (2006) Optimal design of experiments. Siam, Philadelphia

Rees DH (1967) Some designs of use in serology. Biometrics 23:779-791

Shah KR, Sinha BK (1989) Theory of optimal designs. Springer, New York

The GAP Group (2014) GAP_-groups, algorithms, and programming, Version 4.7.4. http://www.gapsystem.org

Publisher's Note Springer Nature remains neutral with regard to jurisdictional claims in published maps and institutional affiliations. 